\title{
FAULT DETECTION OF CRACKED BEAMS LIKE STRUCTURE USING ARTIFICIAL NEURAL NETWORK (ANN) APPROACH
}

\author{
Rabinarayan Sethi ${ }^{1}$, D.R.K. Parhi' ${ }^{2}$, S.K.Senapati ${ }^{3}$ \\ ${ }^{1,3}$ Department of Mechanical Engineering, Indira Gandhi Institute of Technology, Sarang, Dhenkanal, Odisha, India. \\ ${ }^{2}$ Department of Mechanical Engineering, National Institute of Technology, Rourkela, 769008, India. \\ rabinsethi@gmail.com
}

\begin{abstract}
This research work presents to train an artificial neural network to damage prediction of the beam with various possible fractured conditions. An experimental investigation has been conducted on the cantilever beam structures to measure the vibration responses, both in the fractured and un-fractured conditions. A study of the dynamic behavior of a damaged and un- damaged conditions beams expose that, there exists a close relationship among the relative natural frequencies at different modes at different relative crack location and depth. The entire data obtain from experimental procedure have been used as the basis to formulate the technique based on Artificial Neural Network (ANN). These outputs of the experimental analysis were mainly used as inputs data for training and analysis the artificial neural network. The Radial basis function $(R B F)$ network implemented using $M A T L A B$ had engaged in this study. The spotlight of this work has been to study the probability of using an artificial neural network trained with only natural frequency data to assess of the damage in fractured cantilever beam. The developed neural network system can predict the location and intensity of the crack in a close proximity to the actual results.
\end{abstract}

Keywords: Vibration, Damage, Relative Natural Frequency, Artificial Neural Network.

\section{INTRODUCTION}

The presence of a fault, in general, in a structure, undermines the viability of the structure and leads to shorter lifetime and opens the way for complete failure of the system. So, development of a programmed method for crack prediction in engineering applications is desirable. The presence of a crack in a mechanical element increases the flexibility, decreases the vibration frequencies and modifies the amplitude of vibration. The each change in vibration signature can be potentially used to locate and intensity of the crack. Hence, it is of concern to design and develop an Artificial intelligence technique based technique for an online crack diagnosis to avoid catastrophic failure of the structural system.

\section{LITERATURE REVIEW}

The artificial neural network (ANN) have used as an effective substitute tool for solving the inverse engineering problems because of the pattern-matching capability. The outcomes of ANN are quite encouraging and prove the robustness of the proposed damage assessment algorithm.

A methodology has been for online structure health monitoring of axially flowing for blades with the use of the neural network. The developed neural network have trained with the extracted vibration features from the experimental test structures ( Oberholster and Heyns 2006). A fault detection inverse algorithm to estimate the fault intensities of joints in truss bridge structure using back propagation neural network method ( Mehrjoo et al. 2008). Neural network method with a combination of vibration and thermal fault detection signatures to develop a fault detection too 1( Agosto et al. 2008). A machine learning algorithm using the artificial neural network for health monitoring of wind turbine blades ( Dervilis et al. 2014). The effectiveness of the proposed method has validated through experimental vibration analysis. The damage diagnosis method basing on Artificial Neural Network (ANN) using frequency response function ( Bandara et al. 2014 ). It has seen that this methodology can predict the crack and its severity with high accuracy. As ANN is not very common to the traditional mechanical fraternity, a detailed description and the working principle of ANN is presented as follows.

\section{EXPERIMENTAL SET UP}

In the present study, for Mild steel beam specimens $(1000 \times 47.5 \times 6)$ with different crack location and different crack depth at ascending order, the natural frequencies for different mode shapes were calculated. The complete experimental set-up has been as shown in the figure 1 . The experiment has performed on 1st, 2 nd and the 3rd mode of vibration with the help of the exciter. Using FRF Measurement in Pulse 11 version software, natural frequencies have recorded on the vibration indicator. Results of the amplitude of transverse vibration obtained from the experiment at different locations along the length of the beam have been recorded by placing the vibration pick-up where the corresponding resonant frequencies have tuned to the vibration generator. 


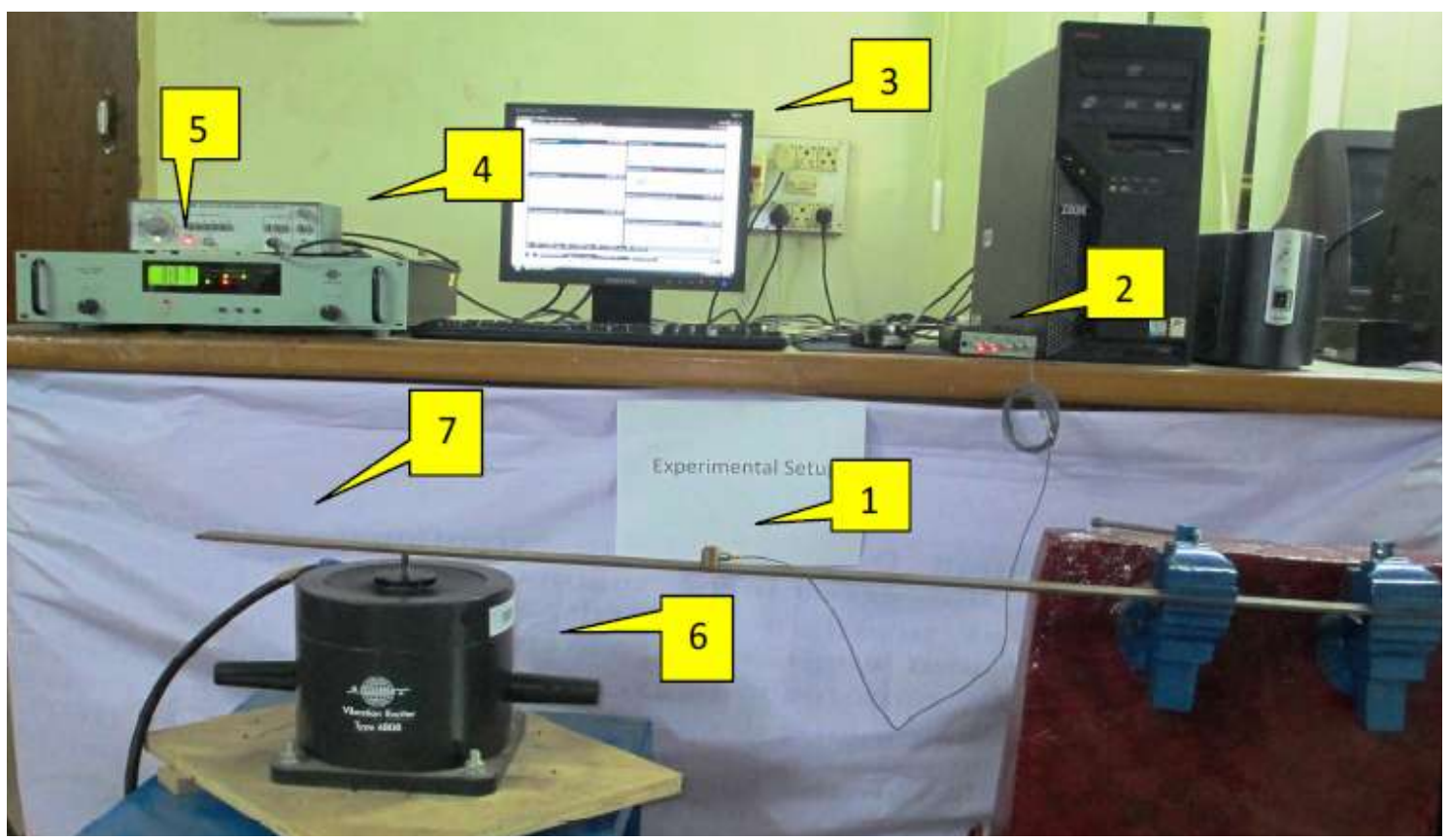

Figure 1: Experimental Setup 1. Vibration Pick-up, 2. Vibration analyzer, 3. Vibration indicator, 4. Function generator, 5.Power amplifier, 6. Vibration Exciter, 7. Cantilever beam specimen.

The relative natural frequency of various relative crack depths at a different relative crack location at mode 1, 2 and 3 are shown in Figures 3.4, 3.5 and 3.6 obtained from experimental analysis respectively. It has noticed that with an increase the depth in crack depth frequency decreases for all the modes of vibration.Also, it has observed that there are significant variations in relative frequency at the vicinity of crack location due to the presence of a crack. It has seen that the values of the dimensionless compliances increase with the increase of relative crack depth. In the other hand, it can be stated that stiffness of the beam decreases with depth.

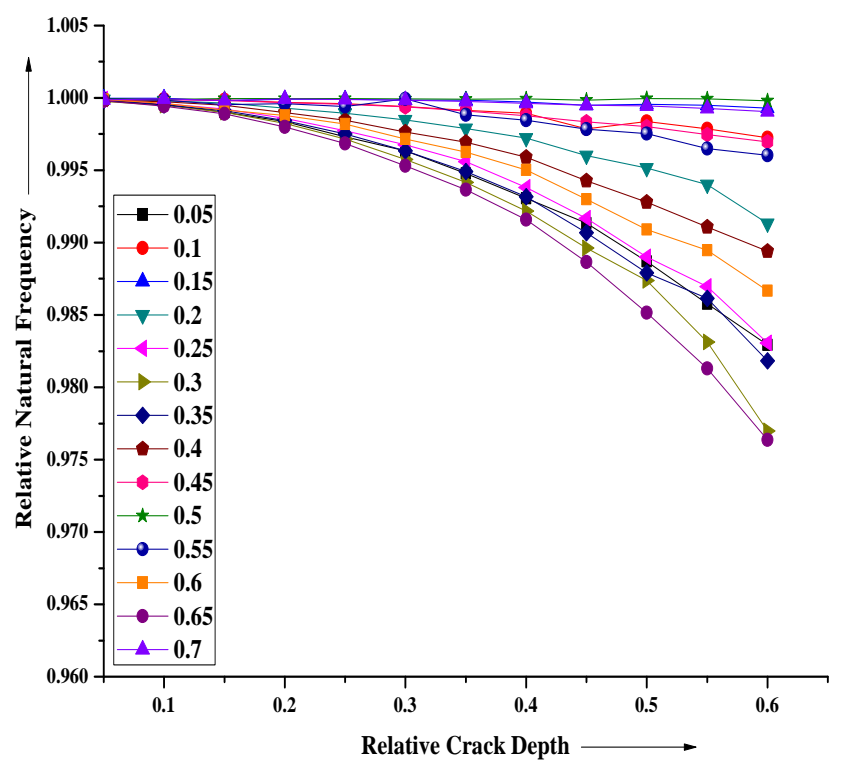

Figure 3. a : RFNF of various RCDs at a different RCLs location at the first mode of vibration .

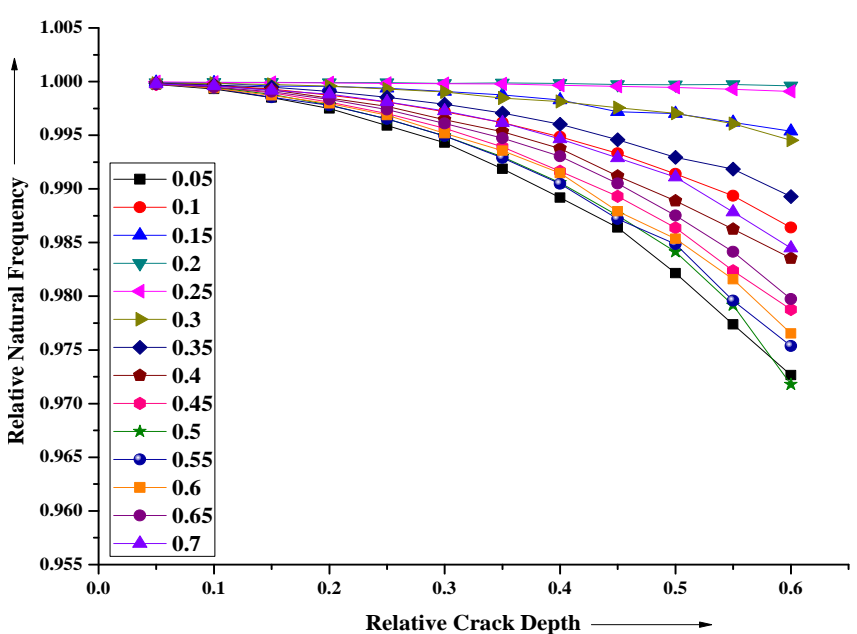

Figure 3. b : RSNF of various RCDs at a different RCLs location at second mode of vibration.

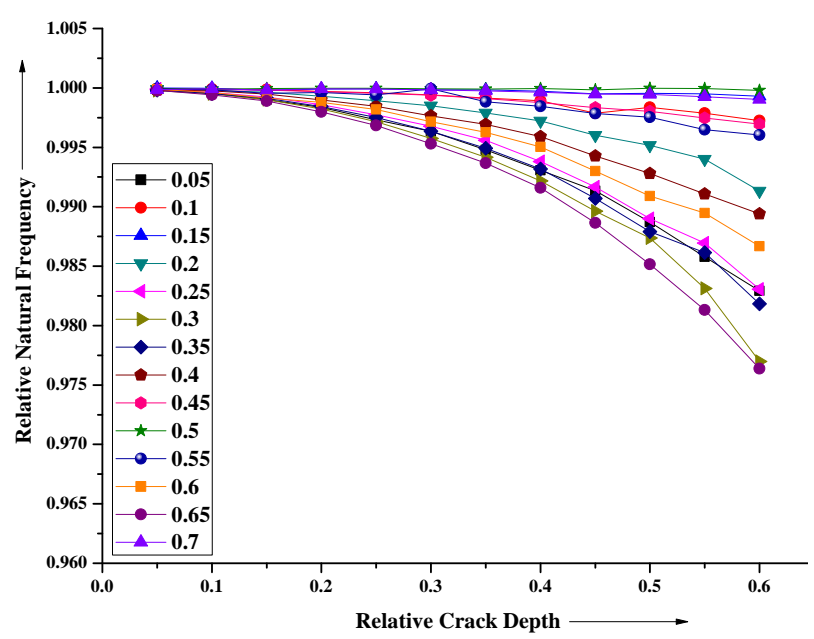

Figure 3. c : RTNF of various RCDs at a different RCLs location at the third mode of vibration. 


\section{ANALYSIS OF NEURAL TECHNIQUE}

The Radial basis Function (RBF) network network technique has been developed for detection of the relative crack location and relative crack depth. The neural network has got three input parameters and two output parameters. The inputs to the neural network are as follows; Relative first natural frequency = "RFNF"; Relative second natural frequency $=$ "RSNF"; Relative third natural frequency = "RTNF". The outputs from the neural network are as follows; Relative crack location $=$ "RCL" and Relative crack depth = "RCD" . The RBF network has also trained basing on experimental data bases. The RBF network has then used to complete the comparative study. The default value of the mean squared error goal set in the RBF network have 0.0, and the spread value have also taken as the default value, which have 1.0. The more the spread, more the smoother the function approximation has obtained.

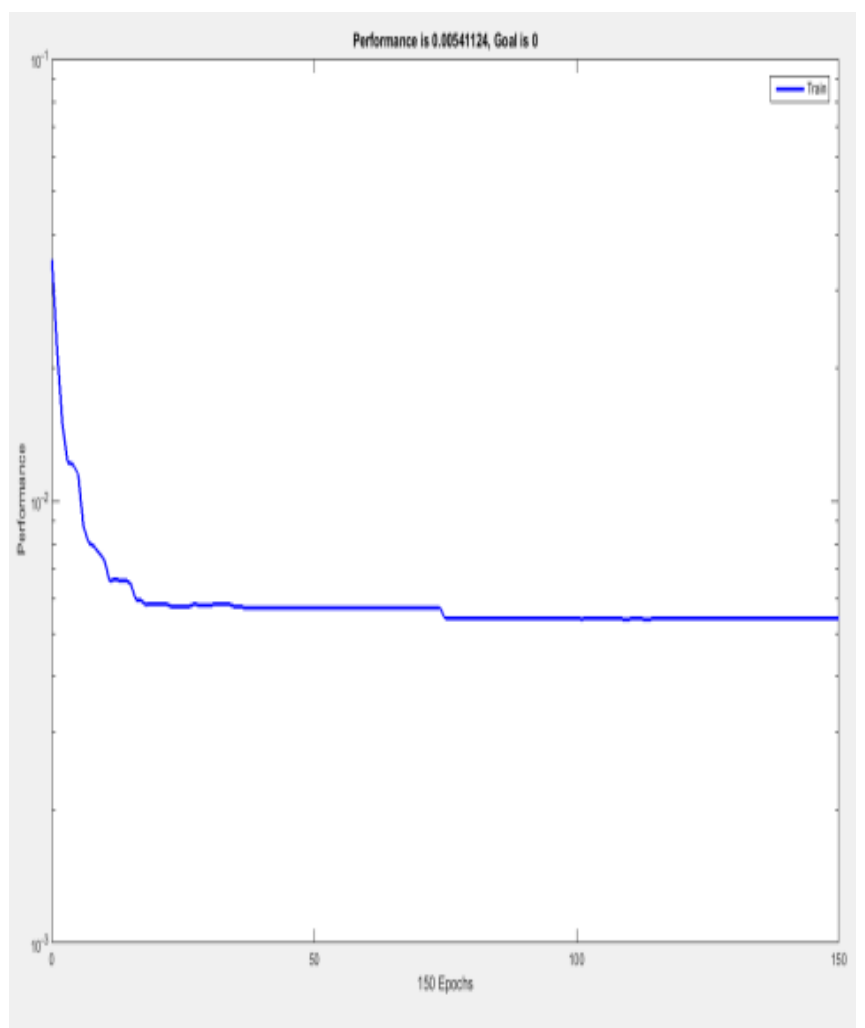

Figure 4 : Performance graph of RBF network.

The performance plot have shown in Figure 4. It has seen that the error remains constant after 180 generations (epochs).

The following comparison of experimental and RBF network results are also shown in Figures 5 (a) and (b), respectively for relative crack depth and relative crack location. It has seen that there has a good agreement between the experimental and predicted values.

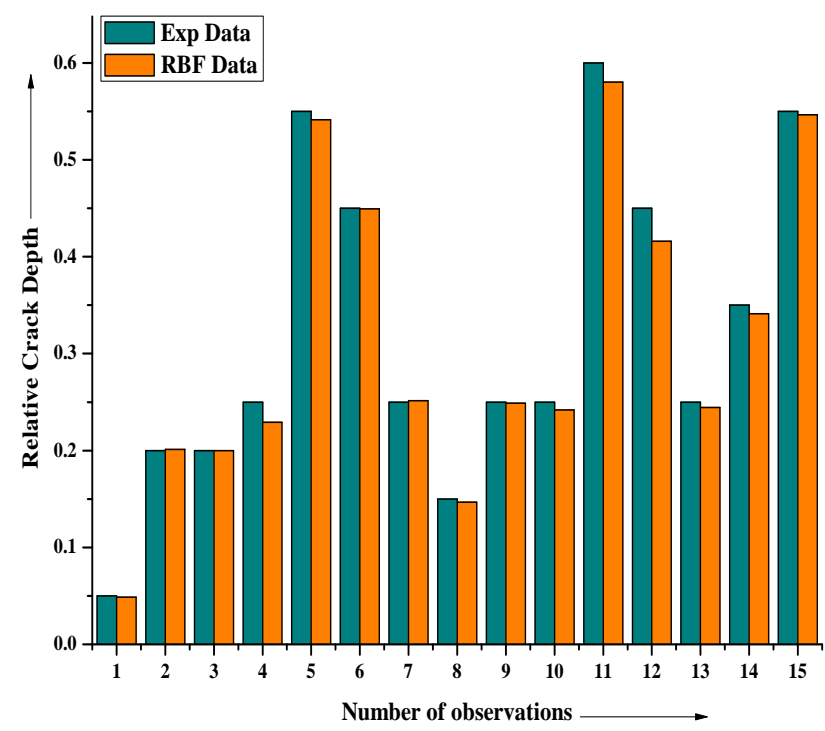

Figure 5 (a) Comparison of results between experimental Data and RBF network controllers for relative crack depth.

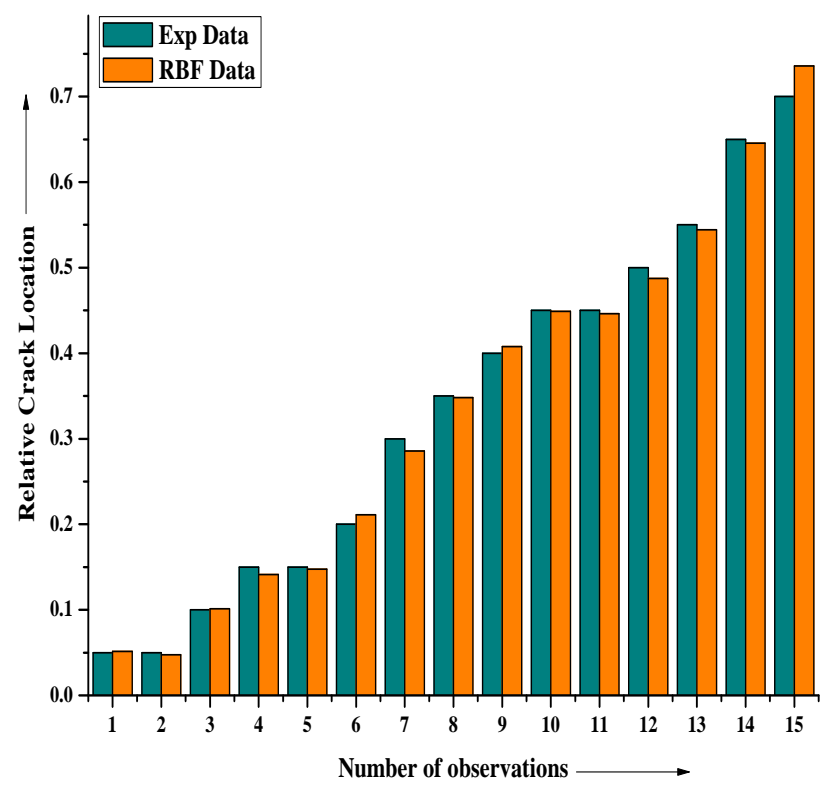

Figure 5 (b) Comparison of results between experimental Data and RBF network controllers for relative crack locations.

\section{DISCUSSION}

The main purpose of this research work has to develop a proficient neural network technique for diagnosis of crack in a vibrating structure. In this analysis, relative natural frequency forms the basis for prediction of crack parameters.

As per the experimental database, in case of RBF function the error is within $1 \%$ to 8 and $0 \%$ to $6 \%$ for relative crack depth and relative crack location respectively. It has observed that the predicted results of neural network technique have reasonably adequate and in agreement with the experimental result. The developed models have efficiently used for crack prediction problems. 


\section{CONCLUSION}

Following conclusions can be drawn on the basis of the results and discussions made from the present investigations. Due to the presence of crack, the beam structure undergoes remarkable changes in natural frequencies. And these changes depend upon the crack location and its intensity. The neural network technique considered here is used to predict the crack location and its intensity by using relative deviation of first three natural frequencies inputs. The neural network predicted results are reasonably acceptable and in agreement with the experimental data. This developed neural network technique can be used as a smart fault detecting tool for different types of vibrating structures. Further research can be made to generate different neural network technique for more efficient fault identification.

\section{REFERENCES}

[1] Agosto F. J., Serrano D. , Shafiq B. and Cecchini A. , "Neural network based nondestructive evaluation of sandwich composites", Composites: Part B, vol. 39, pp. 217-225, 2008.

[2] Bandara R. P., Chan T. H. T. and Thambiratnam D. P., "Frequency response function based damage identification using principal component analysis and pattern recognition technique", Engineering Structures, vol. 66, pp.116-128, 2014.

[3] Dervilis N. , Choi M., Taylor S.G., Barthorpe R.J., Park G., Farrar C.R. and Worden K. , "On damage diagnosis for a wind turbine blade using pattern recognition", Journal of Sound and Vibration, vol. 333, no. 6, pp. $1833-1850,2014$.

[4] Mehrjoo M., Khaji N, Moharrami H. and Bahreininejad A. , "Damage detection of truss bridge joints using Artificial Neural Networks", Expert Systems with Applications, vol. 35, pp. 1122-1131, 2008.

[5] Oberholster J. and Heyns P. S., "On-line fan blade damage detection using neural networks", Mechanical Systems and Signal Processing, vol. 20, pp. 78-93, 2006. 